\title{
PHARMACOLOGICAL EFFECTS OF Sapindus mukorossi
}

\author{
Aparna UPADHYAY \& D.K. SINGH
}

\begin{abstract}
SUMMARY
Sapindus mukorossi is an extremely valuable medicinal plant, distributed in tropical and sub-tropical regions of Asia. The aim of present review is to form a short compilation of the phytochemical composition and pharmacological properties of this multipurpose tree. The main phytoconstituents isolated and identified from different parts of this plant are triterpenoidal saponins of oleanane, dammarane and tirucullane type. The structure and chemical names of all the types of triterpenoidal saponins reported in Sapindus mukorossi are included in this review. Many research studies have been conducted to prove the plant's potential as being spermicidal, contraceptive, hepatoprotective, emetic, anti-inflammatory and anti-protozoal. The present review highlights some of the salient pharmacological uses of Sapindus mukorossi.
\end{abstract}

KEYWORDS: Sapindus mukorossi; Pharmacology; Saponins.

\section{INTRODUCTION}

Sapindus mukorossi Gaertn., a member of the family Sapindaceae, is commonly known by several names such as soapnut, soapberry, washnut, reetha, aritha, dodan and doadni. It is a deciduous tree widely grown in upper reaches of Indo-Gangetic plains, Shivaliks and sub Himalayan tracts at altitudes from $200 \mathrm{~m}$ to $1500 \mathrm{~m}$. The Sapindus mukorossi is a fairly large, deciduous tree with a straight trunk up to 12 meters in height, sometimes attaining a height of $20 \mathrm{~m}$ and a girth of $1.8 \mathrm{~m}$, with a globose crown and rather fine leathery foliage. Bark is dark to pale yellow, fairly smooth, with many vertical lines of lenticels and fine fissures exfoliating in irregular wood scales. The blaze is $0.8-1.3 \mathrm{~cm}$, hard, not fibrous, pale orange brown, brittle and granular. Leaves are $30-50 \mathrm{~cm}$ long, alternate, paripinnate; common petiole very narrowly bordered, glabrous; leaflets 5-10 pairs, opposite or alternate, $5-18$ by $2.5-5 \mathrm{~cm}$, lanceolate, acuminate, entire, glabrous, often slightly falcate or oblique; petioles $2-5 \mathrm{~m}$ long. Inflorescence is a compound terminal panicle, $30 \mathrm{~cm}$ or more in length, with pubescent branches. Flowers are about $5 \mathrm{~mm}$ across, small, terminal, polygamous, greenish white, subsessile, numerous, mostly bisexual. Sepals 5, each with a woolly scale on either side above the claw. Fruits are globose, fleshy, 1-seeded drupe, sometimes two drupels together, about $1.8-2.5 \mathrm{~cm}$ across. Seeds are $0.8-1.3 \mathrm{~cm}$ in diameter, globose, smooth, black and loosely placed in dry fruit ${ }^{7}$.

The fruit is valued for the saponins $(10.1 \%)$ present in the pericarp and constitutes up to $56.5 \%$ of the drupe known for inhibiting tumor cell growth ${ }^{37}$. In China and Japan it has been used as a remedy for centuries. In Japan its pericarp is called "enmei-hi", which means "life prolonging pericarp" and in China "wu-huan-zi", the "non-illness fruit" ${ }^{37}$. The major compounds isolated from Sapindus mukorossi are triterpenoidal saponins of mainly three oleanane, dammarane and tirucullane types. Recently many of the pharmacological actions of this plant have been explored which includes the antimicrobial ${ }^{19}$, cytotoxic $^{29}$, molluscicidal ${ }^{16,42}$, insecticidal ${ }^{12,26}$, piscicidal $^{44}$ and fungicidal ${ }^{37,41}$ activities. One of the most talked about activities of this plant is the contraceptive activity of the saponins extracted from the pericarp of the fruit ${ }^{11,27}$.

Sapindus mukorossi is well known for its folk medicinal values ${ }^{30}$. Pericarps of Sapindus mukorossi have been traditionally used as an expectorant as well as a source of natural surfactant ${ }^{20}$. Due to the presence of saponins, soapnut is well known for its detergent and insecticidal properties and it is traditionally used for removing lice from the scalp. The fruits are of considerable importance for their medicinal value for treating a number of diseases like excessive salivation, pimples, epilepsy, chlorosis, migranes, eczema and psoriasis ${ }^{21}$. The powdered seeds are employed in the treatment of dental caries, arthritis, common colds, constipation and nausea ${ }^{8}$. The seeds of Sapindus mukorossi are used in Ayurvedic medicine to remove tan and freckles from the skin. It cleanses the skin of oily secretion and is even used as a cleanser for washing hair as it forms a rich, natural lather. The leaves are used in baths to relieve joint pain and the roots are used in the treatment of gout and rheumatism. Since ancient times Sapindus mukorossi has been used as a detergent for shawls and silks. The fruit of Sapindus mukorossi was utilized by Indian jewelers for restoring the brightness of tarnished ornaments made of gold, silver and other precious metals ${ }^{33}$. 


\section{PHYTOCHEMICAL CONSTITUENTS}

The major constituents of Sapindus mukorossi fruit are saponins (10\%-11.5\%), sugars (10\%) and mucilage ${ }^{10}$. Saponins are secondary plant metabolites with divergent biological activities ${ }^{10}$. Sapindus saponins are a mixture of six sapindosides (sapindosides A, B, C, D and mukorozi saponins $\left(E_{1}\right.$ and $\left.Y_{1}\right)$, with sapindoside $B$ as one of the major constituents, isolated by n-butanol extraction of the ethanolic extract of fruit pericarp of Sapindus mukorossi and identified by liquid chromatography and mass spectroscopy ${ }^{28}$. Saponins are a large family of structurally-related compounds of steroid or triterpenoid aglycone (sapogenin) linked to one or more oligosaccharide moieties by glycosidic linkage. The aglycone, or sapogenin, may contain one or more unsaturated C-C bonds. The oligosaccharide chain is normally attached at the $\mathrm{C}_{3}$ position (monodesmosidic), but many saponins have an additional sugar moiety at the $\mathrm{C}_{2,6}$ or $\mathrm{C}_{2,8}$ position (bidesmosidic) ${ }^{10}$. The great complexity of the saponin structure arises from the variability of the aglycone structure, the nature of the side chains and the position of attachment of these moieties on the aglycone ${ }^{10}$. The carbohydrate moiety consists of pentoses, hexoses or uronic acids. Due to this complexity, saponins are difficult to classify. Because it is no longer customary to classify compounds based on their physicochemical or biological properties, a state of the art classification based on the biosynthesis of the saponin carbon skeletons was proposed by VINCKEN et $a l .{ }^{43}$.

Different types of triterpene, saponins of oleanane, dammarane and tirucullane type were isolated from the galls, fruits and roots of Sapindus mukorossi. Oleanane type triterpenoid saponins named Sapindoside A\&B (Fig. 34 \& 35) were reported from the fruits of Sapindus mukorossi ${ }^{6}$. Sapindoside C (Fig. 36) ${ }^{4}$, Sapindoside D (Fig. 37) ${ }^{5}$, which is a hexaoside of hederagenin, and Sapindoside E (Fig. 38$)^{3}$, a nonaoside of hederagenin, was isolated and identified from the methanolic extract of the fruits of Sapindus mukorossi.

Dammarane-type saponins, named Sapinmusaponins A \& B (Fig. 11 \& 12), C-E (Fig. 15, 16, 17), together with three known phenylpropanoid glycosides, were isolated from the galls of Sapindus mukorossi $i^{45}$. Tirucallane-type saponins, sapinmusaponins F-J (Fig. 18-22), were isolated from the galls of Sapindus mukorossi as reported by HUANG et al. ${ }^{17}$. The structures of these saponins were elucidated on the basis of spectroscopic analysis including 1D and 2D NMR techniques.

Triterpene saponins of oleanane type like, Sapinmusaponin K-N (Fig. 25-28), Mukorozisaponin G \& E1 (Fig. 29-30), Sapindoside A \& B along with dammarane types like Sapinmusaponin O and P (Fig. 13 \& 14) were isolated from fruits and the galls of Sapindus mukorossi as per HUANG et al. ${ }^{15}$. In another study by NAKAYAMA et al. ${ }^{23}$, Mukorozisaponin Y1 (Fig. 31), Y2 (Fig. 32), X (Fig. 33) were isolated from the pericarp of Sapindus mukorossi.

Fractionation of an ethanolic extract of the galls of Sapindus mukorossi has resulted in the isolation of two tirucallane type triterpenoid saponins, sapinmusaponin Q and R (Fig. 23-24), along with three known oleanane type triterpenoid saponins: sapindoside A, sapindoside $B$, and hederagenin3 -O-[ $\beta$-D-xylopyranosyl- $(1 \rightarrow 3)]-\alpha$-L-rhamnopyranosyl- $(1 \rightarrow 2)+-\alpha-\mathrm{L}$ arabinopyranoside ${ }^{14}$. The roots of Sapindus mukorossi contain tirucallanetype triterpenoid saponins like Sapimukoside A \& B ${ }^{38}$, Sapimukoside C $\& \mathrm{D}^{25}$. Further investigation of the roots of Sapindus mukorossi by NI et al. ${ }^{24}$ reported the presence of Sapimukosides E-J ${ }^{24}$. The structures of Sapimukosides A-J are shown in Fig. 1 to Fig. 10 respectively. Table 1 shows whole view of all the saponins isolated from Sapindus mukorossi.

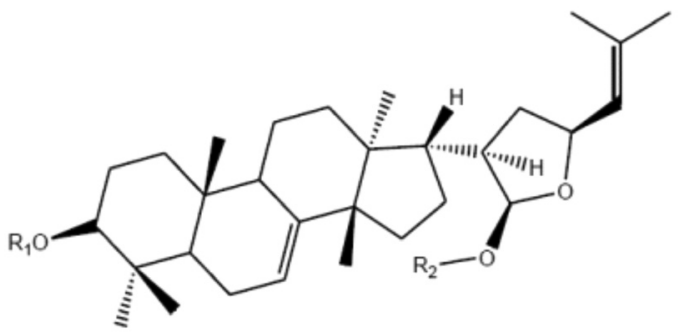

FIG.: STRUCTURE OF SAPIMUKOSIDES A-J

\begin{tabular}{|c|c|c|}
\hline Fig & $\mathbf{R}_{1}$ & $\mathbf{R}_{2}$ \\
\hline & 3-Ara & $\mathrm{H}$ \\
\hline \multirow[t]{2}{*}{1} & Glc & \\
\hline & 2-Rha & \\
\hline \multirow[t]{2}{*}{2} & $\mathrm{Glc}_{6}-\mathrm{Rha}$ & $\mathrm{H}$ \\
\hline & 3-Ara & \\
\hline \multirow[t]{3}{*}{3} & Glc & Et \\
\hline & 2-Rha & \\
\hline & 3-Ara & \\
\hline \multirow[t]{3}{*}{4} & Glc & $\mathrm{Me}$ \\
\hline & 2-Rha & \\
\hline & 3-Ara & \\
\hline \multirow[t]{3}{*}{5} & Glc & Et \\
\hline & 2-Rha - -Ara & \\
\hline & 3-Ara & \\
\hline \multirow[t]{3}{*}{6} & Glc & Et \\
\hline & 2-Rha $-\mathrm{Xyl}$ & \\
\hline & 3-Ara & \\
\hline \multirow[t]{2}{*}{7} & Glc & $\mathrm{Me}$ \\
\hline & 2-Rha $-X y l$ & \\
\hline \multirow[t]{3}{*}{8} & 3-Ara & Et \\
\hline & Glc & \\
\hline & 2-Rha - Ara & \\
\hline \multirow[t]{3}{*}{9} & 3-Rha & $\mathrm{Me}$ \\
\hline & Glc & \\
\hline & 2-Rha3-Ara & \\
\hline 10 & Glc $_{6}-$ Rha Et & \\
\hline
\end{tabular}

Abbreviations

Glc: $\beta$-D-Glucopyranosyl

Rha: $\alpha$-L-hamnopyranosyl

Ara: $\alpha$-L-rabinopyranosyl

Xyl: $\beta$-D-Xylopyranosyl

\section{BIOLOGICAL EFFECTS}

1. Anti-bacterial activity: IBRAHIM et al. ${ }^{19}$ evaluated that ethanolic and chloroform extracts of Sapindus mukorossi inhibited the growth of Helicobacter pylori (both sensitive and resistant), at very low concentrations, when given orally for seven days to male wister rats. In the in vitro study, the isolates show a considerable zone of inhibition at very low concentration $(10 \mu \mathrm{g} / \mathrm{mL})$ and in the in vivo 
Table 1

List of Saponins isolated from Sapindus mukorossi

\begin{tabular}{|c|c|c|c|c|}
\hline Saponins & Chemical name & $\begin{array}{c}\text { Tirucullane/oleanane/ } \\
\text { dammarane type }\end{array}$ & Structure & Reference \\
\hline \multicolumn{5}{|c|}{ Sapindoside } \\
\hline $\mathrm{A}$ & $\begin{array}{l}\text { Hederagenin-3-O- } \alpha \text {-L-arabinosyl- }(2 \rightarrow 1)-\alpha-\mathrm{L}- \\
\text { rhamnopyranoside }\end{array}$ & Oleanane & 34 & Chirva et al., 1970 a \\
\hline $\mathrm{B}$ & $\begin{array}{l}\text { Hederagenin-3-O- } \alpha \text {-L-arabinosyl- }(2 \rightarrow 1)-O-\alpha-\mathrm{L}- \\
\text { rhamnopyranosyl- }(3 \rightarrow 1)-\beta \text {-D-xylanopyranoside }\end{array}$ & Oleanane & 35 & Chirva et al., 1970 a \\
\hline $\mathrm{C}$ & $\begin{array}{l}\text { Hederagenin-3-O- } \beta \text {-D-glucosyl }(1 \rightarrow 4)-\beta \text {-D-xylosyl } \\
(1 \rightarrow 3) \text { - } \alpha \text {-L-rhamnosyl }(1 \rightarrow 2) \text { - } \alpha \text {-L-arabinoside }\end{array}$ & Oleanane & 36 & Chirva et al., $1970 \mathrm{~b}$ \\
\hline \multicolumn{5}{|c|}{ Sapinmusaponin } \\
\hline $\mathrm{A}$ & $\begin{array}{l}\text { 3,7,20(S),22-tetrahydroxydammar-24-ene-3-O- } \alpha \text {-L- } \\
\text { rhamnopyranosyl-( }(1 \rightarrow 2) \text {-D-glucopyranoside }\end{array}$ & Dammarane & 11 & Yao et al., 2005 \\
\hline $\mathrm{B}$ & $\begin{array}{l}\text { 3,7,20(S),22,23-pentahydroxydammar-24-ene-3-O- } \alpha \text {-L- } \\
\text { rhamnopyranosyl- }(1 \rightarrow 2) \text {-D-glucopyranoside }\end{array}$ & Dammarane & 12 & Yao et al., 2005 \\
\hline $\mathrm{C}$ & $\begin{array}{l}3,7,20(S), 22,25 \text {-pentahydroxydammar-23-ene-3-O- } \alpha \text {-L- } \\
\text { rhamnopyranosyl-( }(1 \rightarrow 2) \text {-D-glucopyranoside }\end{array}$ & Dammarane & 15 & Yao et al., 2005 \\
\hline $\mathrm{D}$ & $\begin{array}{l}\text { 25-methoxy-3,7,20(S),22-tetrahydroxydammar-23-ene-3-O- } \alpha \text { - } \\
\text { L-rhamnopyranosyl- }(1 \rightarrow 2) \text {-D-glucopyranoside, }\end{array}$ & Dammarane & 16 & Yao et al., 2005 \\
\hline $\mathrm{E}$ & $\begin{array}{l}\text { 25-methoxy-3,7,20(R)-trihydroxydammar-23-ene-3-O- } \alpha \text {-L- } \\
\text { rhamnopyranosyl- }(1 \rightarrow 2) \text {-D-glucopyranoside }\end{array}$ & Dammarane & 17 & Yao et al., 2005 \\
\hline $\mathrm{F}$ & $\begin{array}{l}21 \beta \text {-methoxy-3- } \beta \text {-21(S), 23I-epoxy tirucall-7,24-diene-3-O- } \\
\alpha \text {-L-rhamnopyranosyl- }(1 \rightarrow 6)-\beta \text {-D-glucopyranosyl }\end{array}$ & Tirucullane & 18 & Huang et al., 2006 \\
\hline G & $\begin{array}{l}21 \alpha \text {-methoxy-3- } \beta \text {-21(S), 23I-epoxy tirucall-7,24-diene-3-O- } \alpha \text { - } \\
\text { L-rhamnopyranosyl- }(1 \rightarrow 6)-\beta \text {-D-glucopyranosyl }\end{array}$ & Tirucullane & 19 & Huang et al., 2006 \\
\hline $\mathrm{H}$ & $\begin{array}{l}21 \alpha \text {-methoxy-3- } \beta \text {-21(S), 23I-epoxy tirucall-7,24-diene-3-O- } \\
\alpha \text {-L-rhamnopyranosyl- }(1 \rightarrow 2)-\beta \text {-D-glucopyranosyl }\end{array}$ & Tirucullane & 20 & Huang et al., 2006 \\
\hline $\mathrm{I}$ & $\begin{array}{l}\text { 21 } \beta \text {-methoxy-3- } \beta \text {-21(S), 23I-epoxy tirucall-7,24-diene-3- } O \text { - } \\
\alpha \text {-L-dirhamnopyranosyl- }(1 \rightarrow 2,6)-\beta \text {-D-glucopyranosyl }\end{array}$ & Tirucullane & 21 & Huang et al., 2006 \\
\hline $\mathrm{J}$ & $\begin{array}{l}\text { 21 } \alpha \text {-methoxy-3- } \beta \text {-21(S), 23I-epoxy tirucall-7,24-diene-3- } O \text { - } \\
\alpha \text {-L-dirhamnopyranosyl- }(1 \rightarrow 2,6)-\beta \text {-D-glucopyranosyl }\end{array}$ & Tirucullane & 22 & Huang et al., 2006 \\
\hline $\mathrm{K}$ & $\begin{array}{l}\text { hederagenin-3-O-(3-O-acetyl-alpha-L-arabinopyranosyl)- } \\
(1 \rightarrow 3) \text {-alpha-L-rhamnopyranosyl- }(1 \rightarrow 2) \text {-alpha-L- } \\
\text { arabinopyranoside }\end{array}$ & Oleanane & 25 & Huang et al., 2008 \\
\hline $\mathrm{L}$ & $\begin{array}{l}\text { hederagenin-3-O-(4-O-acetyl-alpha-L-arabinopyranosyl)- } \\
(1 \rightarrow 3) \text {-alpha-L-rhamnopyranosyl-( } 1 \rightarrow 2) \text {-alpha-L-arabino- } \\
\text { pyranoside, }\end{array}$ & Oleanane & 26 & Huang et al., 2008 \\
\hline M & $\begin{array}{l}\text { hederagenin-3-O-(2,3-O-diacetyl-beta-D-xylopyranosyl)- } \\
(1 \rightarrow 3) \text {-alpha-L-rhamnopyranosyl-( }(\rightarrow 2) \text {-alpha-L- } \\
\text { arabinopyranoside }\end{array}$ & Oleanane & 27 & Huang et al., 2008 \\
\hline $\mathrm{N}$ & $\begin{array}{l}\text { hederagenin-3-O-(2,4-O-diacetyl-beta-D-xylopyranosyl)- } \\
(1 \rightarrow 3) \text {-alpha-L-rhamnopyranosyl- }(1 \rightarrow 2) \text {-alpha-L- } \\
\text { arabinopyranoside }\end{array}$ & Oleanane & 28 & Huang et al., 2008 \\
\hline $\mathrm{O}$ & $\begin{array}{l}\text { 3,7,20(S)-trihydroxydammar-24-ene-3- } O \text {-alpha-L- } \\
\text { rhamnopyranosyl- }(1 \rightarrow 2) \text {-beta-D-glucopyranoside }\end{array}$ & Dammarane & 13 & Huang et al., 2008 \\
\hline $\mathrm{P}$ & $\begin{array}{l}\text { 3,7,20(R)-trihydroxydammar-24-ene-3- } O \text {-alpha-L- } \\
\text { rhamnopyranosyl- }(1 \rightarrow 2) \text {-beta-d-glucopyranoside }\end{array}$ & Dammarane & 14 & Huang et al., 2007 \\
\hline Q & 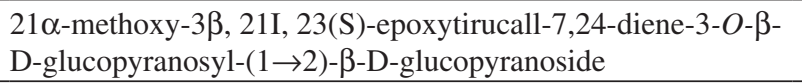 & Tirucullane & 23 & Huang et al., 2007 \\
\hline $\mathrm{R}$ & 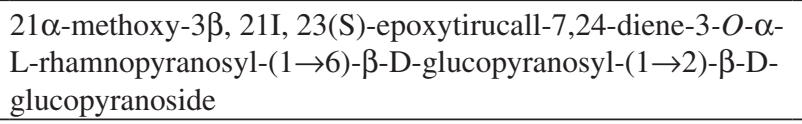 & Tirucullane & 24 & Huang et al., 2007 \\
\hline
\end{tabular}


Table 1

List of Saponins isolated from Sapindus mukorossi (cont.)

\begin{tabular}{|c|c|c|c|c|}
\hline \multicolumn{5}{|c|}{ Sapinmukoside } \\
\hline A & $\begin{array}{l}\text { 3-O- } \alpha \text {-L-rhamnopyranosyl- }(1 \rightarrow 2)-* \alpha-\mathrm{L}- \\
\text { arabinopyranosyl- }(1 \rightarrow 3)+-\beta \text {-D-glucopyranosyl-21, } \\
\text { 23R-epoxyl tirucall-7, 24R-diene-3 } \beta, 2 \text {-diol }\end{array}$ & Tirucullane & 1 & Teng et al., 2003 \\
\hline B & $\begin{array}{l}\text { 3-O- } \alpha \text {-L-rhamnopyranosyl-( } 1 \rightarrow 6)-\beta \text {-D-glucopyranosyl-21, } \\
\text { 23R-epoxyl tirucall-7, 24R-diene-3 } \beta, 21 \text {-diol }\end{array}$ & Tirucullane & 2 & Teng et al., 2003 \\
\hline $\mathrm{C}$ & 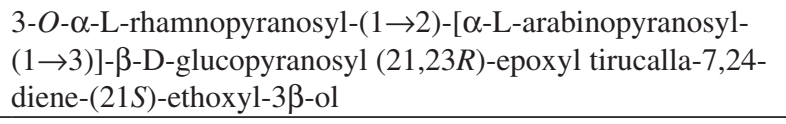 & Tirucullane & 3 & Teng et al., 2004 \\
\hline $\mathrm{D}$ & 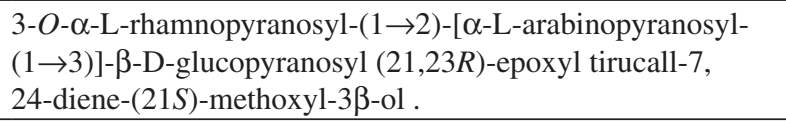 & Tirucullane & 4 & Teng et al., 2004 \\
\hline $\mathrm{E}$ & 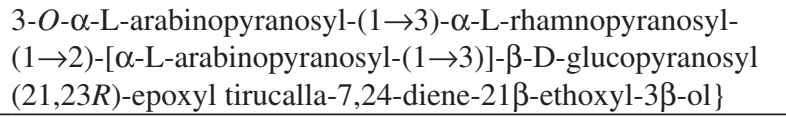 & Tirucullane & 5 & Ni et al., 2006 \\
\hline $\mathrm{F}$ & 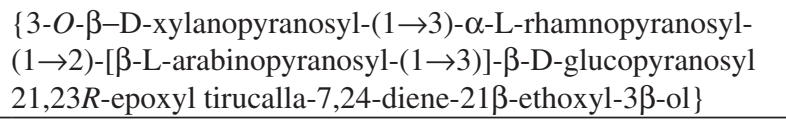 & Tirucullane & 6 & Ni et al., 2006 \\
\hline G & 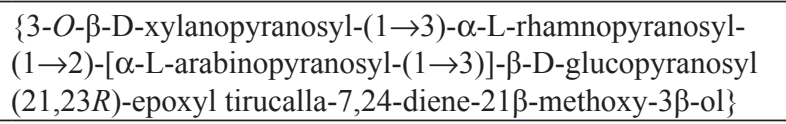 & Tirucullane & 7 & Ni et al., 2006 \\
\hline $\mathrm{H}$ & 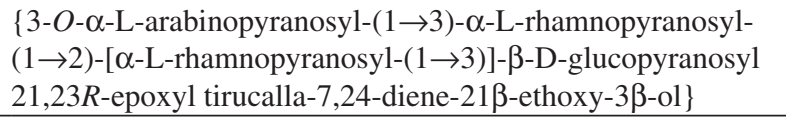 & Tirucullane & 8 & Ni et al., 2006 \\
\hline I & 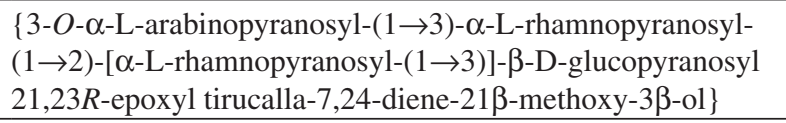 & Tirucullane & 9 & Ni et al., 2006 \\
\hline $\mathrm{J}$ & 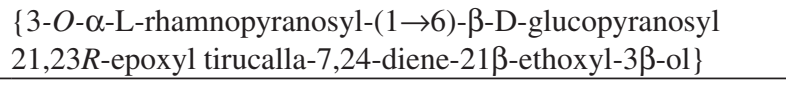 & Tirucullane & 10 & Ni et al., 2006 \\
\hline \multicolumn{5}{|c|}{ Mukorozi-saponin } \\
\hline G & $\begin{array}{l}\text { Hederagenin-3-O-(2-O-acetyl- } \beta \text {-D-xylanopyranosyl)-( }(1 \rightarrow 3) \text { - } \\
\alpha \text {-L-rhamnopyranosyl- }(1 \rightarrow 2)-\alpha \text {-L-arabinoside. }\end{array}$ & Oleanane & 29 & Huang et al., 2008 \\
\hline E1 & $\begin{array}{l}\text { Hederagenin-3- } O \text { - } \alpha \text {-L-arabinosyl- }(1 \rightarrow 3)-\alpha-\mathrm{L}- \\
\text { rhamnopyranosyl- }(1 \rightarrow 2)-\alpha \text {-L-arabinoside. }\end{array}$ & Oleanane & 30 & Huang et al., 2008 \\
\hline
\end{tabular}

study the Helicobacter pylori infection was cleared with minimal dose extracts of $2.5 \mathrm{mg} / \mathrm{mL}$.

2. Insecticidal activity: Saponins possess insecticidal activity, causing mortality and/or growth inhibition in the insects tested, the cotton leafworm Spodoptera littoralis caterpillars and the pea aphid Acyrthosiphon pisum. In the experiments with Acyrthosiphon pisum, $0.1 \%$ saponin killed all aphids, whereas with Spodoptera some caterpillars were still able to develop into apparently normal adults on food containing 7\% saponin ${ }^{12}$. Saponins can be employed as novel natural tactics in integrated pest management (IPM) to control pest insects, which fit in modern agriculture and horticulture ${ }^{13}$. Ethanolic extract of Sapindus mukorossi was investigated for repellency and insecticidal activity against Sitophilus oryzae and Pediculus humanus. Average mortality percentage indicated that the extracts caused significant mortality and repellency on the target insects and bioassays indicated that toxic and repellent effect was proportional to the concentration ${ }^{26}$.
3. Spermicidal activity: Saponins from Sapindus mukorossi are known to be spermicidal ${ }^{11,27}$. Morphological changes in human ejaculated spermatozoa after exposure to this saponin were evaluated under scanning electron microscopy. The minimum effective concentration $(0.05 \%$ in spot test) did not affect the surface topography after exposure for one minute. However, incubation of spermatozoa for 10 minutes resulted in extensive vesiculation and a disruption of the plasma membrane in the head region. Higher concentrations $(0.1 \%, 1.25 \%, 2.5 \%$ and $5.0 \%)$ caused more or less similar changes which included vesiculation, vacuolation, disruption or erosion of membranes in the head region. These findings suggest that the morphological changes observed are due to alterations in the glycoproteins associated with the lipid bilayer of the plasma membrane of spermatozoa ${ }^{8}$. This spermicidal property has been used in contraceptive cream ${ }^{9}$.

4. Anti-Trichomonas activity: TIWARI et al. ${ }^{39}$ demonstrated that the Sapindus saponin mixture shows anti-Trichomonas activity at a 10 -fold lower concentration $(0.005 \%)$ than its minimal effective 


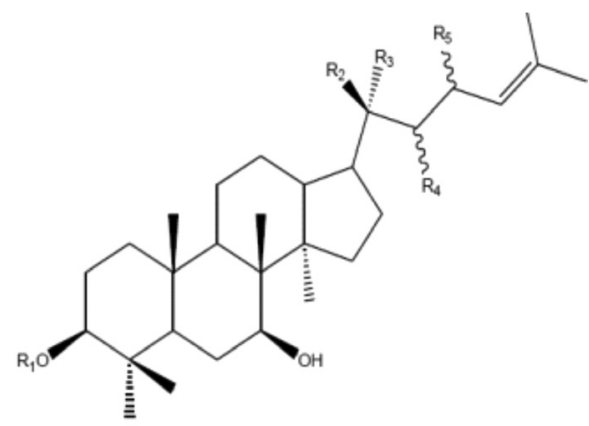

FIG.: STRUCTURE OF SAPIMUSAPONINS A-B AND O-P

\begin{tabular}{lccccc}
\hline Fig & $\mathbf{R}_{\mathbf{1}}$ & $\mathbf{R}_{\mathbf{2}}$ & $\mathbf{R}_{\mathbf{3}}$ & $\mathbf{R}_{\mathbf{4}}$ & $\mathbf{R}_{\mathbf{5}}$ \\
\hline $\mathbf{1 1}$ & $\mathrm{Glc}_{2}-\mathrm{Rha}$ & $\mathrm{H}$ & $\mathrm{OH}$ & $\mathrm{OH}$ & $\mathrm{H}$ \\
$\mathbf{1 2}$ & $\mathrm{Glc}_{2}-\mathrm{Rha}$ & $\mathrm{H}$ & $\mathrm{OH}$ & $\mathrm{OH}$ & $\mathrm{OH}$ \\
$\mathbf{1 3}$ & $\mathrm{Glc}_{2}-\mathrm{Rha}$ & $\mathrm{OH}$ & $\mathrm{CH}_{3}$ & $\mathrm{H}$ & $\mathrm{H}$ \\
$\mathbf{1 4}$ & $\mathrm{Glc}_{2}-\mathrm{Rha}$ & $\mathrm{CH}_{3}$ & $\mathrm{OH}$ & $\mathrm{H}$ & $\mathrm{H}$ \\
\hline
\end{tabular}

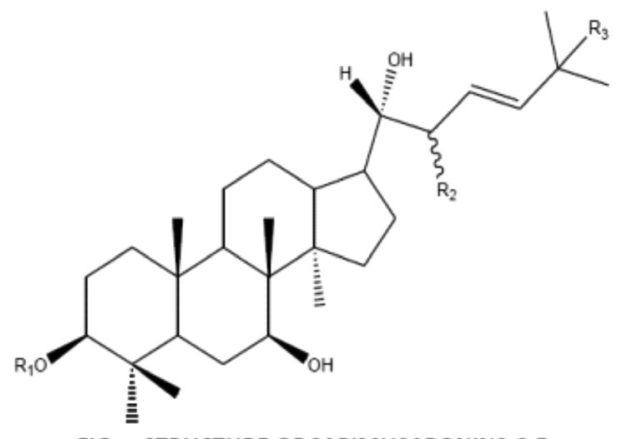

FIG.: STRUCTURE OF SAPIMUSAPONINS C-E

\begin{tabular}{lccc}
\hline Fig & $\mathbf{R}_{\mathbf{1}}$ & $\mathbf{R}_{\mathbf{2}}$ & $\mathbf{R}_{\mathbf{3}}$ \\
\hline $\mathbf{1 5}$ & $\mathrm{Glc}_{2}-\mathrm{Rha}$ & $\mathrm{OH}$ & $\mathrm{OH}$ \\
$\mathbf{1 6}$ & $\mathrm{Glc}_{2}-\mathrm{Rha}$ & $\mathrm{OH}$ & $\mathrm{OCH}_{3}$ \\
$\mathbf{1 7}$ & $\mathrm{Glc}_{2}-\mathrm{Rha}$ & $\mathrm{H}$ & $\mathrm{OCH}_{3}$ \\
\hline
\end{tabular}

spermicidal concentration against human spermatozoa $(0.05 \%)^{11}$. Saponin concentration dependently inhibited the ability of parasites to adhere to HeLa cells and decreased the proteolytic activity of the parasite's cysteine proteinases. This was associated with the decreased expression of adhesin AP65 and membrane-expressed cysteine proteinase TvCP2 genes. Saponins produced no adverse effect on host cells in the mitochondrial reduction potential measurement assay. Saponin disrupts the actin cytoskeleton network beneath the cell membrane and affects membrane-mediated adherence of Trichomonas to the host cells.

5. Anti-cancer activity: Due to the great variability in saponin structure, saponins always display anti-tumorigenic effect through varieties of anti-tumor pathways. There are more than 11 distinguished classes of saponins including dammaranes, tirucallanes, lupanes, hopanes, oleananes, taraxasteranes, ursanes, cycloartanes, lanostanes, cucurbitanes and steroids. Ginsenosides, belonging to dammaranes, have been found beneficial in the inhibition of tumor angiogenesis by suppressing its inducer in the endothelial cells of blood vessels, and then in the prevention of adhering, invasion and metastasis of tumor cells ${ }^{22}$.

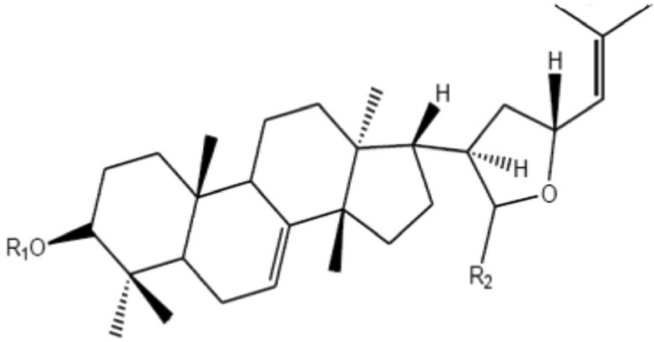

FIG.: STRUCTURE OF SAPIMUSAPONINS F-J, Q-R

\begin{tabular}{lcc}
\hline Fig & $\mathbf{R}_{\mathbf{1}}$ & $\mathbf{R}_{\mathbf{2}}$ \\
$\mathbf{1 8}$ & $\mathrm{Glc}_{6}-\mathrm{Rha}$ & $\beta-\mathrm{OCH}_{3}$ \\
$\mathbf{2 0}$ & $\mathrm{Glc}_{6}-\mathrm{Rha}$ & $\alpha-\mathrm{OCH}_{3}$ \\
& $\mathrm{Glc}_{2}-\mathrm{Rha}$ & $\alpha-\mathrm{OCH}_{3}$ \\
$\mathbf{2 1}$ & 2-Rha & \\
& Glc & $\beta-\mathrm{OCH}_{3}$ \\
& 6-Rha & \\
$\mathbf{2 2}$ & 2-Rha & $\alpha-\mathrm{OCH}_{3}$ \\
$\mathbf{2 3}$ & 6lc & \\
& Glcha & $\alpha-\mathrm{OCH}_{3}$ \\
$\mathbf{2 4}$ & 2-Glc & $\alpha-\mathrm{OCH}_{3}$ \\
& Glc & \\
\hline
\end{tabular}

Dioscin, one of the steroidal saponins, and its aglycone diosgenin also has an extensive anti-tumor effect by cell cycle arrest and apoptosis ${ }^{22}$. The preliminary bioassay data revealed that saponins [39,4]-43] showed moderate cytotoxic activity $\left(\mathrm{ED}_{50} \sim 9-18 \mu \mathrm{g} / \mathrm{mL}\right)$ against human tumor cell lines (Hepa59T/VGH, NCL, HeLa and Med) ${ }^{29}$. Strychnopentamine was the reference compound used in the study. All saponins were reported to be at least five times less active than the reference compound ${ }^{36}$.

6. Hepatoprotective activity: IBRAHIM et al. ${ }^{18}$ reported that the extracts of Sapindus mukorossi $(2.5 \mathrm{mg} / \mathrm{L})$ and Rheum emodi $(3.0 \mathrm{mg} / \mathrm{L})$ have a protective capacity both in vitro on primary hepatocytes cultures and in vivo in a rat model of tetrachloride carbon $\left(\mathrm{CCl}_{4}\right)$ mediated liver injury as judged from serum marker enzyme activities. These cultures were treated with $\mathrm{CCl}_{4}$ and extracts of Sapindus mukorossi \& Rheum emodi. A protective activity could be demonstrated in the $\mathrm{CCl}_{4}$ damaged primary monolayer culture. For the in vivo study, the hepatoprotective capacity of the extract of the fruit pericarp of $S$. mukorossi and the rhizomes of Rheum emodi was analyzed in liver injured $\mathrm{CCl}_{4}$ - treated male rats. Extracts of the fruit pericarp of Sapindus mukorossi $(2.5 \mathrm{mg} /$ $\mathrm{mL})$ and rhizomes of Rheum emodi $(3.0 \mathrm{mg} / \mathrm{mL})$ were found to have protective properties in rats with $\mathrm{CCl}_{4}$ induced liver damage as judged from serum marker enzyme activities. Thus, it was concluded that the extracts of Sapindus mukorossi and Rheum emodi do have a protective capacity both in vitro on primary hepatocytes cultures and in in vivo in a rat model of $\mathrm{CCl}_{4}$ mediated liver injury.

7. Anxiolytic activity: Methanolic extracts of Sapindus mukorossi (200 and $40 \mathrm{mg} / \mathrm{L}$ ) show significant anxiolytic activity as compared to standard anxiolytics Diazepam $(2 \mathrm{mg} / \mathrm{Kg})$ and Fluoxetine $(10 \mathrm{mg} / \mathrm{Kg})^{1}$. 


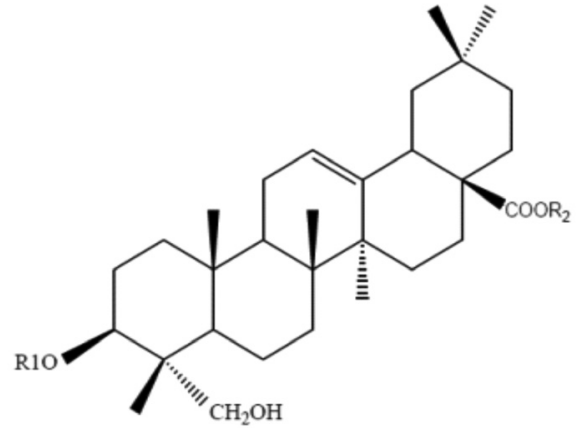

FIG.: STRUCTURE OF SAPIMUSAPONINS K-N, SAPINDOSIDES AE, MUKOROZI SAPONIN E1, G, Y1, Y2 \&X

\begin{tabular}{|c|c|c|}
\hline Fig & $\mathbf{R}_{1}$ & $\mathbf{R}_{2}$ \\
\hline 25 & $\mathrm{Ara}_{2}-\mathrm{Rha}_{3}-\mathrm{Ara}_{3}-\mathrm{OAC}$ & $\mathrm{H}$ \\
\hline \multirow[t]{2}{*}{26} & $\mathrm{Ara}_{2}-\mathrm{Rha}_{3}-\mathrm{Rha}_{4}-\mathrm{OAC}$ & $\mathrm{H}$ \\
\hline & $2-\mathrm{OAC}$ & \\
\hline \multirow[t]{3}{*}{27} & $\mathrm{Ara}_{2}-\mathrm{Rha}_{3}-\mathrm{Xyl}$ & $\mathrm{H}$ \\
\hline & 3-OAC & \\
\hline & 2-OAC & \\
\hline \multirow[t]{3}{*}{28} & $\mathrm{Ara}_{2}-\mathrm{Rha}_{3}-\mathrm{Xyl}$ & $\mathrm{H}$ \\
\hline & 4-OAC & \\
\hline & 3-OAC & \\
\hline \multirow[t]{2}{*}{29} & $\mathrm{Ara}_{2}-\mathrm{Rha}_{3}-\mathrm{Xy}$ & $\mathrm{H}$ \\
\hline & 4-OAC & \\
\hline 30 & $\mathrm{Ara}_{2}-\mathrm{Rha}_{3}-\mathrm{Xyl}_{4}-\mathrm{OAC}$ & $\mathrm{H}$ \\
\hline 31 & $\mathrm{Ara}_{2}-\mathrm{Rha}_{3}-\mathrm{Xyl}$ & $\mathrm{Glc}_{2}-\mathrm{Glc}$ \\
\hline 32 & $\mathrm{Ara}_{2}-\mathrm{Rha}_{3}-\mathrm{Xyl}$ & $\mathrm{Glc}_{2}-\mathrm{Glc}$ \\
\hline 33 & $\mathrm{Ara}_{2}-\mathrm{Rha}$ & $\mathrm{Glc}_{2}-\mathrm{Glc}$ \\
\hline 34 & $\mathrm{Ara}_{2}-\mathrm{Rha}$ & $\mathrm{H}$ \\
\hline 35 & $\mathrm{Ara}_{2}-\mathrm{Rha}_{3}-\mathrm{Xyl}$ & $\mathrm{H}$ \\
\hline \multirow[t]{2}{*}{36} & $\mathrm{Ara}_{2}-\mathrm{Rha}_{3}-\mathrm{Xyl}_{4}-\mathrm{Glc}$ & $\mathrm{H}$ \\
\hline & 6-Rha & \\
\hline \multirow[t]{3}{*}{37} & $\mathrm{Ara}_{2}-\mathrm{Rha}_{3}-\mathrm{Xyl}_{4}-\mathrm{Glc}$ & $\mathrm{H}$ \\
\hline & 2-Glc & \\
\hline & & 6-Rha \\
\hline \multirow[t]{2}{*}{38} & $\mathrm{Ara}_{2}-\mathrm{Rha}_{3}-\mathrm{Xyl}$ & $\mathrm{Ara}_{2}-\mathrm{Rha}_{3}-\mathrm{Xyl}_{4}-\mathrm{Glc}$ \\
\hline & & 2-Glc \\
\hline
\end{tabular}

8. Molluscicidal activity: Extracts of Sapindus mukorossi showed molluscicidal effect against the golden apple snail, Pomacea canaliculata Lamarck. (Ampullariidae) with $\mathrm{LC}_{50}$ values of 85, 22 and $17 \mathrm{ppm}$ at 24,48 and $72 \mathrm{~h}$ exposure period, respectively ${ }^{16}$. Bioassay-directed fractionation of Sapindus mukorossi resulted in the isolation of one new hederagenin-based acetylated saponin, hederagenin 3-O-(2,4-O-diacetyl- $\alpha$-l-arabinopyranoside)-( $1 \rightarrow 3)$ - $\alpha$-L-rhamnopyranosyl-( $(\rightarrow 2)-\alpha$ L-arabinopyranoside [1] , along with six known hederagenin saponins, hederagenin 3-O-(3,4-O-di-acetyl- $\alpha$-L-arabinopyranoside)-( $1 \rightarrow 3)-\alpha$-Lrhamnopyranosyl- $(1 \rightarrow 2)-\alpha$-L-arabinopyranoside [2], hederagenin 3- $O-(3-$ $O$-acetyl- $\beta$-D-xylopyranosyl)-( $1 \rightarrow 3)-\alpha$-L-rhamnopyranosyl- $(1 \rightarrow 2)-\alpha$-Larabinopyranoside [3], hederagenin 3-O-(4-O-acetyl- $\beta$-D-xylopyranosyl)$(1 \rightarrow 3)$ - $\alpha$-L-rhamnopyranosyl-( $1 \rightarrow 2)$ - $\alpha$-L-arabinopyranoside [4], hederagenin 3- $O$-(3,4- $O$-di-acetyl- $\beta$-D-xylopyranosyl)-( $1 \rightarrow 3)-\alpha-\mathrm{L}$ rhamnopyranosyl- $(1 \rightarrow 2)-\alpha$-L-arabinopyranoside [5], hederagenin 3 - $O$ - $\beta$-D-xylopyranosyl- $(1 \rightarrow 3)-\alpha$-L-rhamnopyranosyl- $(1 \rightarrow 2)-\alpha-\mathrm{L}$ arabinopyranoside [6], and hederagenin 3-O- $\alpha$-L-arabinopyranoside [7]. The bioassay data revealed that 1-7 were molluscicidal, causing 70-100\% mortality at $10 \mathrm{ppm}$ against the golden apple snail ${ }^{16}$.

UPADHYAY \& $\mathrm{SINGH}^{42}$ reported that Sapindus mukorossi fruit pericarp is a potential source of botanical molluscicides against Lymnaea acuminata. These snails are the intermediate host of liver fluke Fasciola gigantica, which causes $94 \%$ fascioliasis in the buffalo population of northern India ${ }^{32}$. The active molluscicidal component of Sapindus mukorossi fruit is soluble in chloroform, ether, acetone and ethanol. The toxicity of ethanolic extract of Sapindus mukorossi fruit powder is higher than other extracts which indicates that the molluscicidal component present is more soluble in ethanol than other organic solvents. UPADHYAY \& SINGH $^{42}$ characterized that saponin is the active component present in Sapindus mukorossi fruit by High Performance Liquid Chromatography. A comparison of the molluscicidal activity of the column-purified fraction of Sapindus mukorossi fruit powder with synthetic molluscicides clearly demonstrates that the purified fraction of Sapindus mukorossi is more potent. The $\mathrm{LC}_{50}$ at $96 \mathrm{~h}$ of the columnpurified fraction of Sapindus mukorossi fruit powder $(5.43 \mathrm{mg} / \mathrm{L})$ against Lymnaea acuminata is lower than those of synthetic molluscicidescarbaryl $(14.40 \mathrm{mg} / \mathrm{L})$, phorate $(15.0 \mathrm{mg} / \mathrm{L})$, formothion $(8.56 \mathrm{mg} / \mathrm{L})$ and niclosamide $(11.8 \mathrm{mg} / \mathrm{L})^{32} . \mathrm{LC}_{50}$ at $96 \mathrm{~h}$ of crude powder of Sapindus mukorossi (119.57 mg/L) against Lymnaea acuminata is lower than the crude powder of Canna indica root $(359.02 \mathrm{mg} / \mathrm{L})^{40}$, Thuja orientalis leaf powder $(250.55 \mathrm{mg} / \mathrm{L})$, Thuja orientalis fruit powder $(255.12$ $\mathrm{mg} / \mathrm{L})^{31}$, Zingiber officinale rhizome $(273.80 \mathrm{mg} / \mathrm{L})$, Allium cepa bulb $(253.27 \mathrm{mg} / \mathrm{L})^{34}$.

9. Tyrosinase inhibition and free radical scavenging: CHEN et $a .^{2}{ }^{2}$ first evaluated that the extracts of Sapindus mukorossi seeds using methanol $(\mathrm{MeOH})$, ethyl acetate $(\mathrm{EA})$ or hexane as solvents show tyrosinase inhibition, free radical scavenging, antimicrobial and anticancer properties. Sapindus mukorossi extracts showed strong specific inhibition activities on the proliferation of human melanoma and lung cell lines. The data exhibited the high potential of applying Sapindus mukorossi extracts in medical cosmetology, food supplementation, antibiotics and chemotherapy.

10. Fungicidal activity: The crude extract of Sapindus mukorossi exhibits a strong growth inhibition against the pathogenic yeast Candida albicans, which causes cutaneous candidiasis. Extracts from the dried pericarp of Sapindus L. (Sapindaceae) fruits were investigated for their antifungal activity against clinical isolates of yeasts Candida albicans and Candida non-albicans from vaginal secretions of women with Vulvovaginal Candidiasis. Four clinical isolates of C. albicans, a single clinical isolate of each of the species $C$. parapsilosis, $C$. glabrata, $C$. tropicalis, and the strain of $C$. albicans ATCC 90028 were used. The hydroalcoholic extract was bioactivity-directed against a clinical isolate of $C$. parapsilosis, and showed strong activity. The $\mathrm{n}-\mathrm{BuOH}$ extract and one fraction showed strong activity against all isolates tested ${ }^{41}$. The saponin fraction inhibited the dermatophytic fungi Trichophyton rubrum, Trichophyton mentagrophytes, Sabouraudites canis and Epidermophyton floccosum ${ }^{37}$. 
11. Anti-inflammatory activity: TAKAGI et al. ${ }^{35}$ reported that crude saponin and hederagenin isolated from Sapindus mukorossi inhibited the development of carrageen-induced edema in the rat hind paw as well as on granuloma and exudates formations induced by croton oil in rats. The effects of these agents on vascular permeability and acetic acid induced writhing in mice were also examined. Anti-inflammatory activity on carrageenin edema was observed after intraperitoneal and oral administration of crude saponin, whilst hederagenin and the other agents showed activity only when administered.

12. Piscicidal activity: Effects of Sapindus mukorossi have been studied on fish. Pericarp of Sapindus mukorossi is the most toxic parts yielding $100 \%$ mortality within 12 hours and mean survival time was found to be 1.18 hours. $\mathrm{LD}_{10}, \mathrm{LD}_{50}, \mathrm{LD}_{100}$ ranging between $3.5 \mathrm{ppm}$ and $10 \mathrm{ppm}$ at $48 \mathrm{hrs}$ and possess high potential for fish eradication. Sapindus mukorossi fruit pericarp can be used as a selective eradicant for horny fish like Heteropneustes fossils and channa punctuate ${ }^{44}$.

13 Anti-platelet aggregation activity: HUANG and co workers demonstrated that five new tirucallane type saponins, sapinmusasaponins from the galls of Sapindus mukorossi, showed moderate activity in a 12-0-tetradecanoylphorbol-13-acetate (TPA)-induced Epstein-Barr virus early antigen (EBV-EA) activation assay ${ }^{25}$.

\section{DISCUSSION}

Sapindus mukorossi is a versatile and exceptionally valuable medicinal plant. It is known by such regional names as soapnut, soapberry, washnut, reetha and dodan. The phytochemical screening of the plant extract showed the presence of saponins (10.1\%) present in the pericarp of the fruit. The use of Sapindus mukorossi in folk medicine worldwide ${ }^{30}$ is validated by scientific studies that have demonstrated the efficacy of the extracts in various experimental models. Pharmacological effects of Sapindus mukorossi have been reported like anti-bacterial ${ }^{19}$, insecticidal $^{12,13,26}$, spermicidal $^{11,27}$, anti-trichomonas ${ }^{11,39}$, anti-tumor ${ }^{22,29,36}$, hepatoprotective $^{18}$, anxiolytic ${ }^{1}$, molluscicidal ${ }^{16,42}$, fungicidal ${ }^{37,41}$, antiinflammatory ${ }^{35}$ and piscicidal ${ }^{44}$ activities and are being employed for the treatment of different ailments in the indigenous system of medicine. Although a number of phytochemicals present in Sapindus mukorossi have been isolated and identified by researchers working in different laboratories, their pharmacological/biological studies in human welfare has not been studied so far.

Most of the scientific study is confined to the elaboration of traditional practices of Sapindus mukorossi. There is a long list of saponins present in Sapindus mukorossi. It needs individual attention so that they can be explored in different pharmacological studies. The literature reviewed gives a limited picture of pharmacological effects of Sapindus mukorossi. There is a need for much additional research regarding pharmacological effects of Sapindus mukorossi at molecular level to explain their mode of action.

\section{CONCLUSION}

Sapindus mukorossi is a tropical tree whose numerous economic applications and whose facility of propagation are arousing international interest. It needs to be widely cultivated in most of the areas where climatic conditions favor its optimum growth. In this way, a maximum yield of its different usable parts could be achieved to derive the maximal amount of commodities of a multifarious nature for the welfare of mankind. This plant has been used as traditional medicine for various ailments. The earlier reports on chemical investigation and pharmacological evaluation showed that Sapindus mukorossi contains a number of bio-active novel compounds. As literature illustrates, many biological and pharmacological activities are shown by fractions of crude extracts and isolated substances. Furthermore, the detailed chemical analysis is required to isolate bio-active constituents from Sapindus mukorossi and to trace out their biological activities. Thus, it can be concluded that Sapindus mukorossi can play an important role in modern medical system in near future.

\section{RESUMO}

\section{Efeitos farmacológicos do Sapindus mukorossi}

Sapindus mukorossi é planta medicinal extremamente valiosa distribuída nas regiões tropical e subtropical da Ásia. O propósito da presente revisão é uma compilação curta da composição fitoquímica e das propriedades farmacológicas desta árvore que apresenta múltiplos propósitos. O principal fitoconstituinte isolado e identificado das diferentes partes desta planta são as saponinas triterpenoidais do tipo da oleana, damarana e tiruculana. A estrutura e o nome químico de todos os tipos de saponinas triterpenoidais encontrados no Sapindus mukorossi estão incluídos nesta revisão. Muitas pesquisas tem sido conduzidas para provar o potencial desta planta como espermaticida, contraceptivo, hépato-protetor, emético, anti-inflamatório e anti-protozoário. A presente revisão exalta alguns principais usos farmacológicos do Sapindus mukorossi.

\section{ACKNOWLEDGEMENT}

One of the authors Aparna Upadhyay is thankful to Department of Science and Technology, New Delhi for financial assistance (Inspire Fellowship number- IF10296).

\section{REFERENCES}

1. Chakraborty A, Amudha P, Geetha M, Surjit Singh N. Evaluation of anxiolytic activity of methanolic extract of Sapindus mukorossi Gaertn. in mice. Int J Pharm Bio Scie. 2010;1:1-8.

2. Chen CY, Kuo PL, Chen YH, Huang JC, Ho ML, Lin RJ, et al. Tyrosinase inhibition, free radical scavenging, antimicroorganism and anticancer proliferation activities of Sapindus mukorossi extracts. J Taiwan Inst Chem Engrs. 2010;41:129.

3. Chirva V, Kintya PK, Sosnovskii VA. Triterpene glycosides of Sapindus mukorossi. V. The structure of sapindoside E. Chem Nat Compounds. 1970;6:440-2.

4. Chirva V, Kintya PK, Sosnovskii VA. Triterpene glycosides of Sapindus mukorossi. III. The structure of sapindoside C. Chem Nat Compounds. 1970;6:380-1.

5. Chirva V, Kintya PK, Sosnovskii VA, Zolotarev BM. Triterpene glycosides of Sapindus mukorossi. IV. The structure of sapindoside D. Chem Nat Compounds. 1970;6:316-8.

6. Chirva V, Kintya P, Sosnovskii VA, Krivenchuk PE, Zykova NY. Triterpene glycosides of Sapindus mukorossi. II. The structure of Sapindoside A \& B. Chem Nat Compounds. 1970;6:213-5

7. Chopra R, Ghosh S. Poisonous plants of India. Delhi: The Manager of Publishers; 1946. p. 308. 
8. Dhar JP, Bajpai VK, Setty BS, Kamboj VP. Morphological changes in human spermatozoa as examined under scanning electron microscope after in vitro exposure to saponins isolated from Sapindus mukorossi. Contraception. 1989;39:563-8.

9. Dwivedi AK, Chaudhry M, Sarin JPS. Standardization of a new spermicidal agent Sapindus saponin and its estimation in its formulation. Indian J Pharm Sci. 1990;52:165-7

10. Francis G, Kerem Z, Makkar H, Becker K. The biological action of saponins in animal systems: a review. Br J Nutr. 2002;88:587-605.

11. Garg S, Taluja V, Upadhyay M, Talwar GP. Studies on contraceptive efficacy of Praneem polyherbal cream. Contraception. 1993;48:591-6.

12. Geyter ED, Geelen D, Smagghe G. First results on the insecticidal action of saponins. Commun Agric Appl Biol Sci. 2007;72:645-8.

13. Geyter ED, Lambert E, Geelen D, Smagghe G. Novel advances with plant saponins as natural insecticides to control pest insects. Pest Tech. 2007;1:96-105.

14. Huang HC, Tsai WJ, Liaw CC, Wu SH, Wu YC, Kuo YH. Anti-platelet aggregation triterpene saponins from the galls of Sapindus mukorossi. Chem Pharm Bull (Tokyo). 2007;55:1412-5.

15. Huang HC, Wu MD, Tsai WJ, Liao SC, Liaw CC, Hsu LC, et al. Triterpenoid saponins from the fruits and galls of Sapindus mukorossi. Phytochemistry. 2008;69:1609-16.

16. Huang HC, Liao SC, Chang FR, Kuo YH, Wu YC. Molluscicidal saponins from Sapindus mukorossi, inhibitory agents of Golden Apple snails Pomacea canaliculata. J Agric Food Chem. 2003;51:4916-9.

17. Huang HC, Tsai WJ, Morris-Natschke SL, Tokuda H, Lee KH, Wu YC, et al. Sapinmusaponins F-J, bioactive tirucallane-type saponins from the galls of Sapindus mukorossi. J Nat Prod. 2006;69:763-7.

18. Ibrahim M, Khaja MN, Aara A, Khan AA, Habeeb MA, Devi, YP, et al. Hepatoprotective activity of Sapindus mukorossi and Rheum modi extracts: in vitro and in vivo studies. World J Gastroenterol. 2008;14:2566-71.

19. Ibrahim M, Khan AA, Tiwari SK, Habeeb MA, Khaja MN, Habibullah CM. Anti-microbial activity of Sapindus mukorossi and Rheum modi extracts against Helicobacter pylori: in vitro and in vivo studies. World J Gastroenterol. 2006;12:713642.

20. Kasai R, Fujino H, Kuzuki T, Wong WH, Goto C, Yata N, et al. Acyclic sesquiterpene oligoglycosides from pericarps of Sapindus mukorossi. Phytochemistry. 1986;25:8716.

21. Kirtikar KR, Basu BD. Indian medicinal plants. Allahabad: B.L.M. Publication; 1991.

22. Man S, Gao W, Zhang Y, Huang L, Liu C. Chemical study and medical application of saponins as anti-cancer agents. Fitoterapia. 2010;81:703-14.

23. Nakayama K, Fujino H, Kasai R, Mitoma Y, Yata N, Tanaka O. Solubilizing properties of saponins from Sapindus mukorossi Gaertn. Chem Pharm Bull (Tokyo). $1986 ; 34: 3279-83$

24. Ni W, Hua Y, Liu HY, Teng RW, Kong YC, Hu XY, et al. Tirucallane-type triterpenoid saponins from the roots of Sapindus mukorossi. Chem Pharm Bull (Tokyo). 2006;54:1443-6.

25. Ni W, Hua Y, Teng RW, Kong YC, Chen CX. New tirucallane-type triterpenoid saponins from Sapindus mukorossi. J Asian Nat Prod Res. 2004;6:205-9.

26. Rahman SS, Rahman M, Begum SA, Khan MMR, Bhuiyan MH. Investigation of Sapindus mukorossi extracts for repellency, insecticidal activity and plant growth regulatory effect. J Appl Sci Res. 2007;3:95-101.

27. Rastogi RP, Mehrotra BN. Compendium of Indian medicinal plants. New Delhi: CDRI Publication; 1999. p. 609-10.
28. Saxena D, Pal R, Dwivedi AK, Singh S. Characterization of sapindosides in Sapindus mukorossi saponin (Reetha saponin) and quantitative determination of sapindoside B. J Sci Ind Res. 2004;63:181-6.

29. Sengupta A, Basu SP, Saha S. Triglyceride composition of Sapindus mukorossi seed oil. Lipids. 1975;10:33-40.

30. Sharma A, Sati SC, Sati OP, Sati D, Maneesha Kothiyal SK. Chemical constituents and bioactivities of genus Sapindus. Int J Res Ayurveda Pharm. 2011;2:403-9.

31. Singh A, Singh VK. Molluscicidal activity of Saraca asoca and Thuja orientalis against the fresh water snail Lymnaea acuminata. Vet Parasitol. 2009;164:206-10.

32. Singh DK, Agarwal RA. In vivo and in vitro studies on synergism with anticholinesterase pesticides in the snail Lymnaea acuminata. Arch Environ Contain Toxicol. 1983;12:483-7.

33. Singh N, Kaur A, Yadav K. A reliable in vitro protocol for rapid mass propagation of Sapindus mukorossi Gaertn. Nat Sci. 2010;8:41-7.

34. Singh S, Singh VK, Singh DK. Molluscicidal activity of some common spice plants. Biol. Agric. Hortic. 1997;14:237-49.

35. Takagi K, Park EH, Kato H. Anti-inflammatory activities of hederagenin and crude saponin isolated from Sapindus mukorossi Gaertn. Chem Pharm Bull(Tokyo). 1980;28:1183-8.

36. Takechi M, Tanaka Y. Structure-activity relationships of the saponin $\alpha$-hederin Phytochemistry. 1990;29:451-2.

37. Tanaka O, Tamura Y, Masuda H, Mizutani K. Application of saponins in food and cosmetics: saponins of Mohova Yucca and Sapindus mukorossi Gaertn, saponins used in food and agriculture. New York: Plenum Press; Waller GR and Yamasaki K; 1996. p.1-11.

38. Teng RW, Ni W, Hau Y, Chen CX. Two new tirucallane-type triterpenoid saponins from Sapindus mukorossi. Acta Botanica Sinica. 2003;45:369-72. [Article in Chinese].

39. Tiwari P, Singh D, Singh MM. Anti-Trichomonas activity of Sapindus saponins, a candidate for development as microbicidal contraceptive. J Antimicrob Chemother. 2008;62:526-34.

40. Tripathi SM, Singh DK. Molluscicidal activity of Punica granatum bark, Canna indica root. Braz J Med Biol Res. 2000;33:1351-5.

41. Tsuzuki JK, Svidzinski TIE, Shinobu CS, Silva LFA, Rodrigues-Filho E, Cortex DAG, et al. Antifungal activity of the extracts and saponins from Sapindus saponaria L. An Acad Bras Cienc. 2007;79:577-83.

42. Upadhyay A, Singh DK. Molluscicidal activity of Sapindus mukorossi and Terminalia chebula against the freshwater snail Lymnaea acuminata. Chemosphere. 2011;83:46874.

43. Vincken JP, Heng L, de Groot A, Gruppen H. Saponins, classification and occurrence in the plant kingdom. Phytochemistry. 2007;68:275-97.

44. Virdi, GS. The piscicidal properties of Acorus calamus, Sapindus mukorossi and Xeromphis spinosa on 7 species of fishes of North India. Indian J Phys Nat Sci. 1982;2:28-35.

45. Yao HK, Hui CH, Li-Ming YK, Ya-Wen H, Kuo-Hsiung L, Fang-Rong C, et al. New dammarane-type saponins from the galls of Sapindus mukorossi. J Agric Food Chem. 2005;53:4722 -7.

Received: 17 December 2011

Accepted: 21 March 2012 